\title{
Mechanism of meningeal invasion by Neisseria meningitidis Xavier Nassif
}

\author{
Address: Université Paris Descartes, Faculté de Médecine, site Necker, and INSERM U570, Paris, France
}

Email: Xavier Nassif - xavier.nassif@inserm.fr

from Infectious diseases of the nervous system: pathogenesis and worldwide impact

Paris, France. 10-13 September 2008

Published: 23 September 2008

BMC Proceedings 2008, 2(Suppl I):S32

This abstract is available from: http://www.biomedcentral.com/I753-656I/2/SI/S32

(c) 2008 Nassif; licensee BioMed Central Ltd.

Neisseria meningitidis $(\mathrm{Nm})$ is one of the few pathogens able to cross the BBB and invade the meninges. Compared to other extra cellular bacterial pathogens capable of surviving in the bloodstream, $\mathrm{Nm}$ interact with the endothelial cells of both the brain parenchyma and the meninges. This interaction, which is mediated by type IV pili, requires a transient slow down of the blood flow and is believed to allow the meningeal invasion. Meningococcal adhesion triggers a signalling leading to the formation of cortical plaques. These cortical plaques are enriched in ERM proteins (Ezrin, Moesin, Radixin), cortactin, actin, ErbB2 and adhesion molecules like ICAM-1/2 and VCAM1 . This signal leads to the formation of membrane protrusions. Early work using epithelial cells suggested that $\mathrm{Nm}$ was capable of crossing a monolayer of tight junction forming cells using the transcellular route. However the lack of appropriate in vitro models of human blood brain barrier (BBB) did not allow determining the consequence of $\mathrm{Nm}$ interaction on brain endothelial cells. Using a welldifferentiated human brain endothelial cell line hCMEC/ D3, which closely mimics the $\mathrm{BBB}$, we studied how meningococcal adhesion modifies the integrity of the junctional complexes in brain endothelial cells. We demonstrate that a bacterial pathogen like $\mathrm{Nm}$, recruits the polarity complex Par3/Par6/PKC $\zeta$, in a Cdc42 dependent manner, and hijacks the recruitment of adherens junction proteins. Furthermore we show that this specific targeting requires the previous organization of cortical actin following the recruitment of p120-catenin. Altogether these data suggest that $\mathrm{Nm}$ adhesion onto endothelial cells mimics spot-like adherens junction known to be recruited at nascent cell-cell contacts and is a consequence of a signaling similar to that involved in cell polarization and apical junctional complex assembly. This redistribution of the adherens junction molecules is likely to allow $\mathrm{Nm}$ to cross the $\mathrm{BBB}$ using the paracellular route. 